\title{
Form Factors in the Quark Resonance Model
}

\author{
Krzysztof M. Graczyk* and Jan T. Sobczyk \\ Institute of Theoretical Physics, University of Wroctaw, pl. M. Borna 9, 50-204, Wroctaw, Poland
}

(Dated: April 17, 2018)

\begin{abstract}
Vector and axial form factors in the quark resonance model are analyzed with a combination of theoretical and phenomenological arguments. The new form of form factors is deduced from $\Delta(1232)$ excitation models and available data. The vector part is shown to agree with the resonant contribution to electron-proton inclusive $F_{2}$ data. The axial part is obtained by finding a simultaneous fit to ANL and BNL $\frac{d \sigma}{d Q^{2}}$ neutrino scattering data. The best fit corresponds to $C_{5}^{A}(0)=0.88$ in the Rarita Schwinger formalism.
\end{abstract}

PACS numbers: 13.15.+g, 25.30.Pt,25.70.Ef, 25.75.Dw

Keywords: single pion production, neutrino-nucleon interaction, Rein-Sehgal model

\section{INTRODUCTION}

New generation of neutrino experiments require better knowledge about neutrino-nucleon/nucleus cross sections. In the future a lot of new information will be obtained from the MINER $\nu \mathrm{A}$ experiment [1] but in the meantime one has to rely on the existing data, theoretical models and information which can be deduced from electron scattering experiments.

In the $1 \mathrm{GeV}$ neutrino energy region an important contribution to the total cross section comes from the single pion production (SPP) channels. The theoretical models which describe SPP reactions are usually phenomenological in nature and their predictive power is limited by the precision of SPP neutrino experiments. The standard description is given in the Rarita Schwinger formalism, with hadronic current expressed in terms of several form factors [2, 3, 4, 5, 6]. Recently new interesting theoretical approaches were proposed by Sato and Lee [7] and Hernandez et al. 8].

Almost all neutrino interactions Monte Carlo (MC) generators of events rely on the Rein-Sehgal (RS) 9, 10, 11] model. The RS model is based on the Feynman-Kislinger-Ravndal (FKR) relativistic quark model with SU(6) symmetry group [12]. It includes contributions from 18 resonances in the invariant hadronic mass region $W<2 \mathrm{GeV}$. The input to the model consist of: vector and axial form factors, the value of the Regge slope, masses and widths of the resonances. The functional forms of vector and axial form factor were deduced by applying the model to elastic electron-nucleon and quasi-elastic neutrino-nucleon reactions. The RS model contains also a prescription how to include a non-resonant background.

In this paper we propose modifications of the FKR/RS model. They do not spoil the integrity of the original description and in particular they leave the same number of free parameters/form factors. The motivation to our investigation comes from the fact that in the MiniBooNE and T2K experiments the neutrino beams are most intensive at the energies $700-800 \mathrm{MeV}$. As the consequence in the inelastic channels the precision of predictions depends mostly on the quality in which the $\Delta(1232)$ excitation region is described with higher resonances becoming less important. This implies that in the RS model the form factors should be chosen in such a way that $\Delta(1232)$ production is described as well as possible. In the original FKR/RS model the form factors are fixed by investigating the elastic and quasi-elastic reactions. Our choice is to look at $\Delta(1232)$ excitation processes. The advantage of our prescription is that we obtain form factors which guarantee better description of the $\Delta(1232)$ excitation region.

In the case of vector form factors we use the recent fits to the $\Delta(1232)$ excitation helicity amplitudes 13 . These fits are consistent with the amplitudes obtained in the MAID model for electro- and photo- production [14]. When applied to the FKR model some information is lost because in the FKR model the electric helicity amplitude vanishes. In order to verify our choice we calculate $F_{2}$ electron-proton structure function with original and new vector form factors and conclude that with new form factors the model is closer to the data. Even better agreement with the data requires inclusion of background Born terms as it is done in the MAID model. In our analysis we investigate the resonance form factors and consequently we focus on the neutrino $\operatorname{SPP}$ channel $\left(\nu+p \rightarrow \mu^{-}+p+\pi^{+}\right)$in which it is known that the non-resonance contribution is small. For this reason we find an agreement with $F_{2}$ data satisfactory.

*Electronic address: kgraczyk@ift.uni.wroc.pl 
We did not make a comparison with electron-neutron data, since they are given in the form of electron-deuterium data and in the analysis it is necessary to eliminate nuclear effects.

In the case of axial form factor we find simultaneous fits to two sets (ANL and BNL) of experimental data 15, 16, 17]. We express our fit for the axial form factor in terms of $C_{A}^{5}\left(Q^{2}\right)$ from the Rarita Schwinger formalism. Then by inverting the reasoning our results can be interpreted as a fit to $C_{A}^{5}\left(Q^{2}\right)$. We consider two options: with $C_{A}^{5}(0)=1.2$ guided by the standard PCAC arguments or with $C_{A}^{5}(0)$ left as a free parameter. In the second case we obtain $C_{A}^{5}(0)=0.88$ and the agreement with the data is much better. We notice that recently many authors addressed the problem of the value of $C_{A}^{5}(0)$. In [8] the introduction of non-resonant background terms in accordance with the chiral symmetry led authors to the conclusion that the best fit to both ANL and BNL data is obtained with $C_{A}^{5}(0) \approx 0.867$. In [18] the lattice QCD results are reported with $\frac{2 C_{A}^{5}(0)}{G_{A}(0)} \approx 1.6$. Computations done in the chiral constituent quark model reported in [19] give rise to $C_{A}^{5}(0) \approx 0.93$. The main difference between our approach and the one proposed in [8] is that we do not consider the non-resonant background. As explained before, we try to avoid the issue of non-resonant background and we discuss only one SPP channel $\nu_{\mu}+p \rightarrow\left(\mu^{-}+\Delta^{++}\right) \rightarrow \mu^{-}+p+\pi^{+}$in which it is known that the non-resonant dynamics is not important. One should remember that above mentioned evaluations of $C_{A}^{5}(0)$ were done under different assumptions about remaining axial form factors and thus do not necessary mean the same. For example in [19] the authors obtain $C_{A}^{3}(0) \approx 0.035, C_{A}^{4}(0) \approx-0.25$. The authors of [8] (as also we do) adopt the Adler model values: $C_{A}^{3}\left(Q^{2}\right)=0$ and $C_{A}^{4}\left(Q^{2}\right)=-\frac{C_{A}^{5}\left(Q^{2}\right)}{4}$.

The plan of our paper is the following. In Sect. 2 we introduce the basic notation and necessary information about the objects (helicity amplitudes) calculated in this paper. Sect. 3 contains our derivation of new form factors. The method is based on the analysis of the existing $\Delta(1232)$ excitation data. Helicity amplitudes are calculated in two formalisms which allow to derive new RS form factors. Sect. 4 contains comparison of our results with electromagnetic $F_{2}$ data for electron-proton scattering and with ANL and BNL neutrino scattering data. We show how new form factors modify total cross sections in charged current (CC) and neutral current (NC) SPP channels.

Throughout this paper we call the discussed model as FKR in the case of electromagnetic interactions and as RS when it is applied to weak interactions.
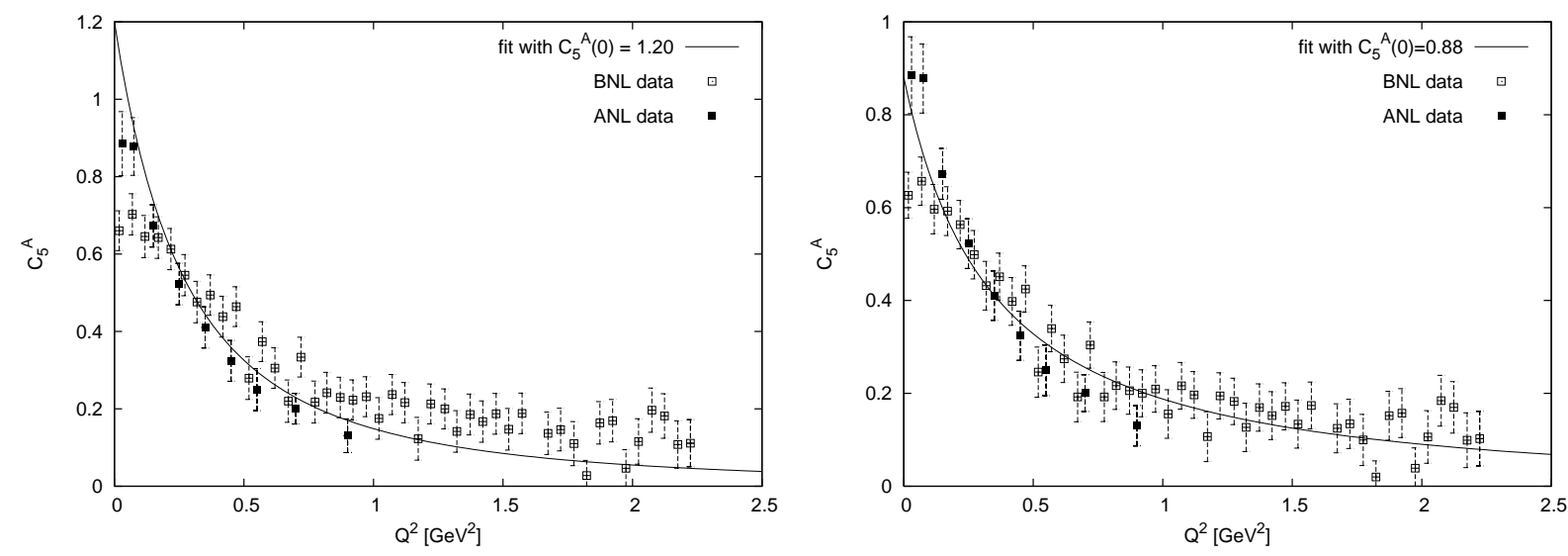

FIG. 1: Fits to $C_{5}^{A}\left(Q^{2}\right)$ with the ANL (black squares) and the BNL (white squares) experimental points. The analytical form of fits are given in Eqs. (52) and (53). The fitting procedure is explained in the text.

\section{FORMALISM}

We consider CC neutrino-production of resonances

$$
\nu_{\mu}(k)+N(p) \longrightarrow \mu^{-}\left(k^{\prime}\right)+\mathcal{N}\left(p^{\prime}\right)
$$

in the framework of the inclusive differential cross section formalism:

$$
\frac{d^{2} \sigma}{d \nu d Q^{2}}=\frac{G^{2} \cos ^{2} \theta_{C}}{8 \pi E^{2}} L_{\mu \nu} W^{\mu \nu}
$$


where

$$
\mathrm{L}_{\mu \nu}=2\left(k^{\prime}{ }_{\mu} k_{\nu}+k_{\mu} k^{\prime}{ }_{\nu}-g_{\mu \nu} k_{\alpha}^{\prime} k^{\alpha}-i \epsilon_{\mu \nu \alpha \beta} k^{\alpha} k^{\prime \beta}\right)
$$

and

$$
W_{\mu \nu}=(2 \pi)^{6} \sum_{\text {final }} \overline{\sum_{s}}\left\langle\text { final }\left|\mathcal{J}_{\mu}^{\text {weak }}(0)\right| p, s\right\rangle\left\langle\text { final }\left|\mathcal{J}_{\nu}^{\text {weak }}(0)\right| p, s\right\rangle^{*} \frac{E_{p}}{M} \delta^{4}\left(p+q-p_{\text {final }}\right)
$$

$k, p, k^{\prime}$ and $p^{\prime}$ denote 4-momenta, the 4-momentum transfer is: $q^{\mu} \equiv k^{\mu}-k^{\prime \mu}=p^{\prime \mu}-p^{\mu}, Q^{2} \equiv-q_{\mu} q^{\mu}, k^{\mu}=(E, \mathbf{k})$, $k^{\prime \mu}=\left(E^{\prime}, \mathbf{k}^{\prime}\right)$ etc. In the LAB frame the axis orientation is chosen so that $q^{\mu}=(\nu, 0,0, q) . M$ denotes the nucleon's and $M_{R}$ 's the resonance mass, $W$ is the invariant hadronic mass of the final state.

We assume that SPP is mediated by the resonance excitation and we focus on the computation of independent helicity amplitudes in the final hadron rest frame:

$$
\begin{aligned}
& f_{+3} \equiv(2 \pi)^{3} \sqrt{\frac{E_{p, \text { res }}}{M}}\left\langle\mathcal{N}, p_{\text {res }}^{\prime}, s^{\prime}=\frac{3}{2}\left|\mathcal{J}_{+}\right| N, p_{\text {res }}, s=\frac{1}{2}\right\rangle, \\
& f_{+1} \equiv(2 \pi)^{3} \sqrt{\frac{E_{p, \text { res }}}{M}}\left\langle\mathcal{N}, p_{\text {res }}^{\prime}, s^{\prime}=\frac{1}{2}\left|\mathcal{J}_{+}\right| N, p_{\text {res }}, s=-\frac{1}{2}\right\rangle, \\
& f_{+0} \equiv(2 \pi)^{3} \sqrt{\frac{E_{p, \text { res }}}{M}}\left\langle\mathcal{N}, p_{\text {res }}^{\prime}, s^{\prime}=\frac{1}{2}\left|\mathcal{J}_{\underline{0}}\right| N, p_{\text {res }}, s=\frac{1}{2}\right\rangle .
\end{aligned}
$$

$p_{\text {res }}, s$ and $p_{\text {res }}^{\prime}, s^{\prime}$ denote momenta and spins of initial $(N)$ and final $(\mathcal{N})$ hadrons in the $\mathcal{N}$ rest frame, and $E_{p, \text { res }}=\sqrt{M^{2}+\vec{p}_{\text {res }}^{2}}$.

The definitions of current operators: $\mathcal{J}_{+}, \mathcal{J}_{-}$and $\mathcal{J}_{\underline{0}}$ are []]:

$$
\mathcal{J}_{ \pm}=\mp \frac{1}{\sqrt{2}}\left(\mathcal{J}_{1} \pm \mathrm{i} \mathcal{J}_{2}\right), \quad \mathcal{J}_{\underline{0}} \equiv \mathcal{J}_{0}+\frac{\nu_{\text {res }}}{q_{\text {res }}} \mathcal{J}_{3} .
$$

Evaluation of the vector part of the current rely on the conserved vector current (CVC) hypothesis and the comparison with the electromagnetic data is required. We use the convention in which electromagnetic current is denoted as $\mathcal{J}_{\nu}^{e m}$ and charged weak current carry no label. Neutral weak current are discussed only occasionally and then the label NC is used.

FKR model is a relativistic harmonic oscillator quark model [12]. Resonance wave functions are constructed based on the SU(6) symmetry [20]. Feynman et al. calculated the hadronic current operators for both electro- and weak CC neutrino-production of the resonances $\mathcal{J}_{\mu}^{e m}, \mathcal{J}_{\mu}$. The NC reactions matrix elements are evaluated with the Standard Model relation [10]:

$$
\mathcal{J}_{\mu}^{N C}=\mathcal{J}_{\mu}^{C C, I_{3}}-2 \sin ^{2} \theta_{W} \mathcal{J}_{\mu}^{e m},
$$

where $\mathcal{J}_{\mu}^{C C, I_{3}}$ is a third component of CC isovector $\mathcal{J}_{\mu}^{C C, I}$.

In the case of the electro-production the current operators are multiplied by the an unknown vector form factor $G_{V}^{R S}$. Similary the axial part is multiplied by the unknown axial form factor $G_{A}^{R S}$ :

$$
\mathcal{J}_{\mu}^{e m} \rightarrow G_{V}^{R S}\left(W, Q^{2}\right) \mathcal{J}_{\mu}^{e m}, \quad \mathcal{J}_{\mu}^{C C}=\mathcal{J}_{\mu}^{V}-\mathcal{J}_{\mu}^{A} \rightarrow G_{V}^{R S}\left(W, Q^{2}\right) \mathcal{J}_{\mu}^{V}-G_{A}^{R S}\left(W, Q^{2}\right) \mathcal{J}_{\mu}^{A},
$$

The original way to calculate $G_{V}^{R S}$ and $G_{A}^{R S}$ was to consider elastic electron-nucleon and quasi-elastic neutrinonucleon scattering [12] (for more details see Appendix A) 99, 21, 22]. In the vector part the results are:

$$
G_{V}^{R S}\left(Q^{2}\right)=G_{D}\left(1+\frac{Q^{2}}{4 M^{2}}\right)^{\frac{1}{2}}, \quad G_{D}=\left(1+\frac{Q^{2}}{M_{V}^{2}}\right)^{-2}
$$

The formulas for the nucleon electric and magnetic form factors calculated in the FKR/RS model are presented in the Tab. III Appendix A. We see that they are reproduced in the approximate way. In the case of proton electric form factor the difference is the extra multiplicative factor $\left(1-\frac{Q^{2}}{2 M^{2}}\right)$. In the case of magnetic form factors the proton and neutron magnetic moments are reproduced with the accuracy of $\sim 5-7 \%$. 
In the FKR/RS model modifications of $G_{V}^{R S}$ for higher level resonances are postulated. New expressions should lead to the above $G_{V}^{R S}$ for $N=0$ (level zero in quark oscillator model) and $W=M$. The following formula was proposed in 23]:

$$
G_{V}^{R S}\left(Q^{2}\right)=G_{D}\left(1+\frac{Q^{2}}{4 W^{2}}\right)^{\frac{1-N}{2}}
$$

This form factor was used to describe the electro-production data. To describe the neutrino-production an alternative form was suggested in $[9]$ :

$$
G_{V}^{R S}\left(Q^{2}\right)=G_{D}\left(1+\frac{Q^{2}}{4 M^{2}}\right)^{\frac{1}{2}-N}
$$

which was also adopted in the original RS model. In [11] it is explained that the first prescription (12) is expected to reproduce both resonant and non-resonant contributions to the inclusive cross section while the second one (13) is aimed to describe only the resonant contribution.

The axial form factor $G_{A}^{R S}$ is reconstructed from the only one nonvanishing axial current helicity amplitude (see Tab. II, Appendix A):

$$
\widetilde{G}_{A}^{R S}=Z G_{A}^{R S}\left(Q^{2}\right)=\frac{3}{5}\left(1+\frac{Q^{2}}{4 M^{2}}\right)^{1 / 2} G_{A}\left(Q^{2}\right),
$$

where quasi-elastic axial form factor is:

$$
G_{A}\left(Q^{2}\right)=1.267\left(1+\frac{Q^{2}}{M_{A}^{2}}\right)^{-2} .
$$

Higher level resonance modifications are again postulated [9, 22] and finally:

$$
\widetilde{G}_{A}^{R S}\left(Q^{2}\right)=0.76\left(1+\frac{Q^{2}}{4 M^{2}}\right)^{\frac{1}{2}-N}\left(1+\frac{Q^{2}}{M_{A}^{2}}\right)^{-2} .
$$

\section{3. $\Delta(1232)$ RESONANCE HELICITY AMPLITUDES.}

\subsection{Vector contribution}

The electromagnetic and weak CC excitation of $\Delta(1232)$ can be modelled using the phenomenological Rarita Schwinger formalism. The vector part of the charged current (up to normalization it is also the electromagnetic current) has a general Lorentz covariant form:

$$
\left\langle\Delta^{++}\left(p^{\prime}\right)\left|\mathcal{J}_{\mu}^{V}\right| N(p)\right\rangle=\sqrt{3} \bar{\Psi}_{\lambda}\left(p^{\prime}\right)\left[g_{\mu}^{\lambda} T_{\nu} q^{\nu}-q^{\lambda} T_{\mu}+g_{\mu}^{\lambda} C_{6}^{V}\right] \gamma_{5} u(p) \frac{1}{(2 \pi)^{3}} \sqrt{\frac{M_{R} M}{E_{p} E_{p^{\prime}}}},
$$

where

$$
T_{\mu}=\frac{C_{3}^{V}}{M} \gamma_{\mu}+\frac{C_{4}^{V}}{M^{2}} p_{\mu}^{\prime}+\frac{C_{5}^{V}}{M^{2}} p_{\mu},
$$

$\Psi_{\lambda}$ is a Rarita-Schwinger field. The conservation of vector current implies:

$$
C_{6}^{V}\left(Q^{2}\right)=0 .
$$

We calculate the helicity amplitudes for the $\Delta(1232)$ production in both RS and Rarita Schwinger formalism.

It is enough to consider three independent amplitudes:

$$
\begin{aligned}
& f_{+3}^{\Delta, V} \equiv(2 \pi)^{3} \sqrt{\frac{E_{p, r e s}}{M}}\left\langle\Delta, p_{\text {res }}^{\prime}, s^{\prime}=\frac{3}{2}\left|\mathcal{J}_{+}^{V}\right| N, p_{\text {res }}, s=\frac{1}{2}\right\rangle, \\
& f_{+1}^{\Delta, V} \equiv(2 \pi)^{3} \sqrt{\frac{E_{p, r e s}}{M}}\left\langle\Delta, p_{\text {res }}^{\prime}, s^{\prime}=\frac{1}{2}\left|\mathcal{J}_{+}^{V}\right| N, p_{\text {res }}, s=-\frac{1}{2}\right\rangle, \\
& f_{+0}^{\Delta, V} \equiv(2 \pi)^{3} \sqrt{\frac{E_{p, r e s}}{M}}\left\langle\Delta, p_{\text {res }}^{\prime}, s^{\prime}=\frac{1}{2}\left|\mathcal{J}_{\underline{0}}^{V}\right| N, p_{\text {res }}, s=\frac{1}{2}\right\rangle .
\end{aligned}
$$



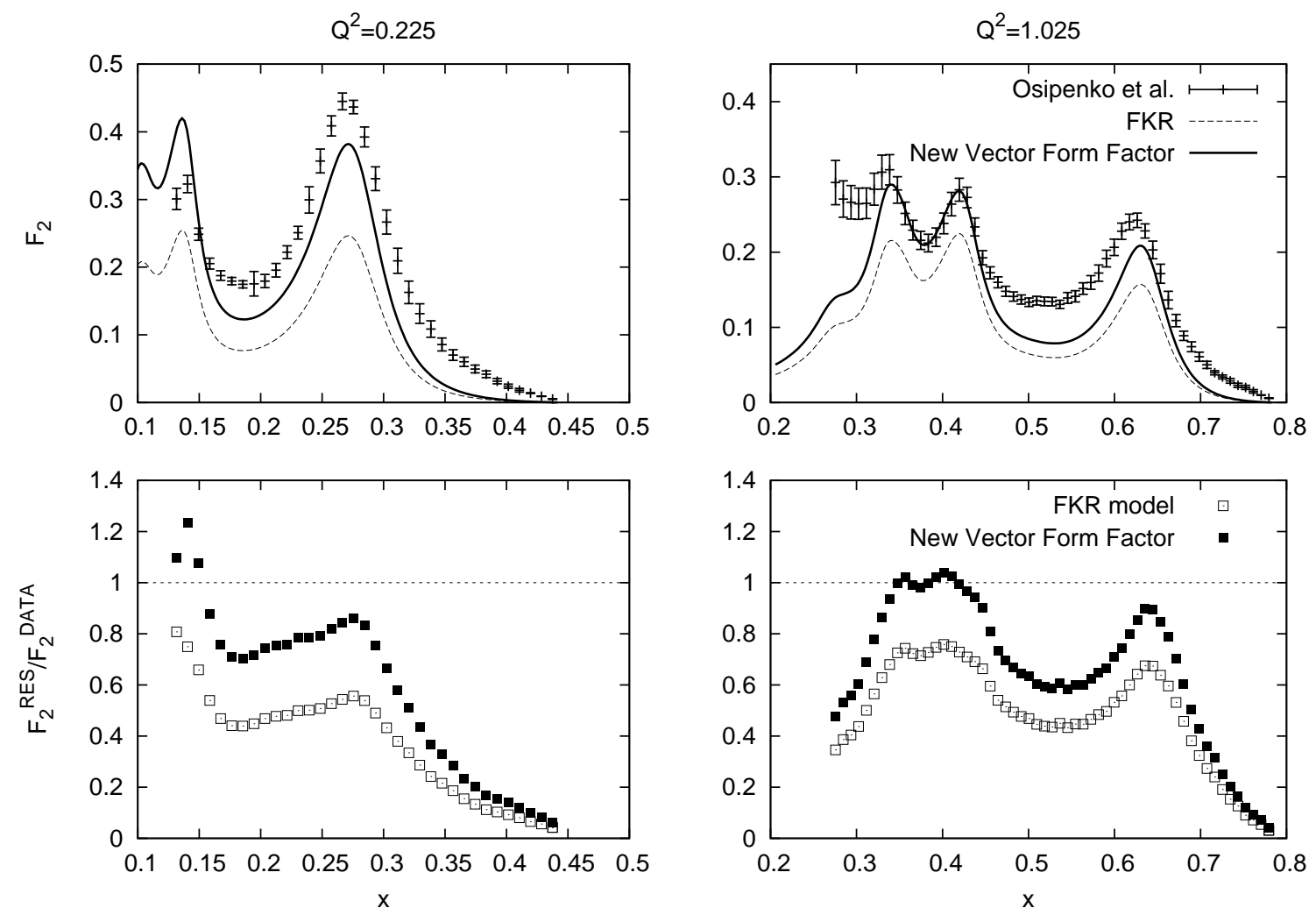

FIG. 2: In the top predictions for $F_{2}$ for $e p$ scattering in the original FKR model and in the model of this paper for $Q^{2}=0.225 \mathrm{GeV}^{2}$ and $Q^{2}=1.025 \mathrm{GeV}^{2}$ are shown. The data is taken from [24]. In the bottom the fractions of the measured strength predicted by both models are presented.

We compare the helicity amplitudes for the vector part of the weak CC current but the relations we get are the same as in the analysis of the helicity amplitudes for the electromagnetic current.

In the Rarita Schwinger formalism we obtain:

$$
\begin{aligned}
f_{+3}^{\Delta, V} & =-N_{q_{r e s}} \frac{q_{\text {res }}}{M+E_{q_{\text {res }}}}\left\{\frac{C_{4}^{V}}{M^{2}} p^{\prime}{ }_{\mu} q^{\mu}+\frac{C_{5}^{V}}{M^{2}} p_{\mu} q^{\mu}+\frac{C_{3}^{V}}{M}(W+M)\right\}, \\
f_{+1}^{\Delta, V} & =\sqrt{\frac{1}{3}} N_{q_{r e s}} \frac{q_{\text {res }}}{M+E_{q_{r e s}}}\left\{\frac{C_{4}^{V}}{M^{2}} p^{\prime}{ }_{\mu} q^{\mu}+\frac{C_{5}^{V}}{M^{2}} p_{\mu} q^{\mu}+\frac{C_{3}^{V}}{M}\left(W+M-2\left(M+E_{q_{\text {res }}}\right)\right)\right\}, \\
f_{+0}^{\Delta, V} & =-\sqrt{\frac{2}{3}} N_{q_{r e s}} \frac{q_{\text {res }}}{M+E_{q_{r e s}}} \sqrt{Q^{2}}\left\{\frac{C_{4}^{V}}{M^{2}} W+\frac{C_{5}^{V}}{M^{2}} \frac{M(M+W)}{W}+\frac{C_{3}^{V}}{M}\right\},
\end{aligned}
$$

where

$$
N_{q_{r e s}} \equiv \sqrt{\frac{M+E_{q_{r e s}}}{2 M}}, \quad E_{q_{r e s}}=\sqrt{M^{2}+q_{r e s}^{2}}
$$

The same expressions for the $\Delta(1232)$ helicity amplitudes were derived before by Lalakulich et. al [13]. In the RS model:

$$
\begin{aligned}
f_{+3}^{\Delta, V, R S} & =-\sqrt{6} \sqrt{\frac{W}{M}} R, \\
f_{+1}^{\Delta, V, R S} & =-\sqrt{2} \sqrt{\frac{W}{M}} R, \\
f_{+0}^{\Delta, V, R S} & =0,
\end{aligned}
$$


where

$$
R \equiv \sqrt{2} \frac{M}{W} \frac{q(M+W)}{Q^{2}+(W+M)^{2}} G_{V}^{R S} .
$$

The equivalence of both models would mean that:

$$
\begin{aligned}
G_{V}^{R S}\left(Q^{2}, W\right) & =\frac{1}{2 \sqrt{3}}\left(1+\frac{Q^{2}}{(M+W)^{2}}\right)^{\frac{1}{2}}\left[C_{4}^{V} \frac{W^{2}-Q^{2}-M^{2}}{2 M^{2}}+C_{5}^{V} \frac{W^{2}+Q^{2}-M^{2}}{2 M^{2}}+\frac{C_{3}^{V}}{M}(W+M)\right], \\
G_{V}^{R S}\left(Q^{2}, W\right) & =-\frac{1}{2 \sqrt{3}}\left(1+\frac{Q^{2}}{(M+W)^{2}}\right)^{\frac{1}{2}}\left[C_{4}^{V} \frac{W^{2}-Q^{2}-M^{2}}{2 M^{2}}+C_{5}^{V} \frac{W^{2}+Q^{2}-M^{2}}{2 M^{2}}-C_{3}^{V} \frac{(M+W) M+Q^{2}}{M W}\right] \\
0 & =C_{4}^{V} \frac{W}{M^{2}}+\frac{C_{5}^{V}}{M} \frac{(M+W)}{W}+\frac{C_{3}^{V}}{M} .
\end{aligned}
$$

In general the above equations with $C_{j}^{V}$ provided by experiment cannot be simultaneously satisfied and solved for $G_{V}^{R S}$. In particular the quark model predicts that electric contribution vanishes (Eq. (27)). The well known exception is the theoretical choice [3]:

$$
C_{5}^{V}=0, \quad C_{3}^{V}=-\frac{W}{M} C_{4}^{V}
$$

This preferred from the point of view of the quark model choice is adopted by many authors. Within this choice there is 1:1 correspondence between $C_{4}^{V}$ and $G_{V}^{R S}[4]$ :

$$
C_{4}^{V}\left(Q^{2}\right)=-4 \sqrt{3}\left(\frac{M}{M+W}\right)^{2}\left(1+\frac{Q^{2}}{(M+W)^{2}}\right)^{-3 / 2} G_{V}^{R S}\left(Q^{2}\right) .
$$

The problem with the choice (28) is that it does not agree well with the existing electromagnetic data. Our strategy is to use the fit to the data proposed in [13]:

$$
\begin{aligned}
& C_{3}^{V}=2.13\left(1+\frac{Q^{2}}{4 M_{V}^{2}}\right)^{-1}\left(1+\frac{Q^{2}}{M_{V}^{2}}\right)^{-2}, \\
& C_{4}^{V}=-1.51\left(1+\frac{Q^{2}}{4 M_{V}^{2}}\right)^{-1}\left(1+\frac{Q^{2}}{M_{V}^{2}}\right)^{-2}, \\
& C_{5}^{V}=0.48\left(1+\frac{Q^{2}}{4 M_{V}^{2}}\right)^{-1}\left(1+\frac{Q^{2}}{0.776 M_{V}^{2}}\right)^{-2}
\end{aligned}
$$

with $M_{V}=0.84 \mathrm{GeV}$ and translate it into the $G_{V}^{R S}$.

With such chosen $C_{j}^{V}$ we cannot reproduce the quark model prediction that the electric contribution vanishes and it is clear that some information has to be lost. In the Rarita Schwinger formalism the current is expressed by three functions and in the RS model by only one. The experimentally measured helicity amplitudes imply that the significance of the electric contribution is on the level of few percent. Since the overall cross section for the pion production has to be supplemented with a non-resonant contribution this drawback is not a very serious one.

We notice that the contributions from $f_{+3}$ and $f_{+1}$ enter the $e p$ cross sections with equal weights. On the other hand, in the FKR model:

$$
f_{+3}^{\Delta, V, F K R} / f_{+1}^{\Delta, V, F K R}=\sqrt{3} .
$$

Therefore, we propose the following vector form factor:

$$
G_{V}^{R S, n e w}\left(W, Q^{2}\right)=\frac{1}{2} \sqrt{3\left(G_{V}^{f_{3}}\left(W, Q^{2}\right)\right)^{2}+\left(G_{V}^{f_{1}}\left(W, Q^{2}\right)\right)^{2}}
$$

where

$$
\begin{aligned}
& G_{V}^{f_{3}}\left(W, Q^{2}\right) \equiv \frac{1}{2 \sqrt{3}}\left(1+\frac{Q^{2}}{(M+W)^{2}}\right)^{\frac{1}{2}}\left[C_{4}^{V} \frac{W^{2}-Q^{2}-M^{2}}{2 M^{2}}+C_{5}^{V} \frac{W^{2}+Q^{2}-M^{2}}{2 M^{2}}+\frac{C_{3}^{V}}{M}(W+M)\right] \\
& G_{V}^{f_{1}}\left(W, Q^{2}\right) \equiv-\frac{1}{2 \sqrt{3}}\left(1+\frac{Q^{2}}{(M+W)^{2}}\right)^{\frac{1}{2}}\left[C_{4}^{V} \frac{W^{2}-Q^{2}-M^{2}}{2 M^{2}}+C_{5}^{V} \frac{W^{2}+Q^{2}-M^{2}}{2 M^{2}}-C_{3}^{V} \frac{(M+W) M+Q^{2}}{M W}\right]
\end{aligned}
$$


We still have to modify $G_{V}^{R S, \text { new }}$ by a factor describing modifications of higher resonance excitations. In the expression for $G_{V}^{R S}$ (13) there is the factor $\left(1+\frac{Q^{2}}{4 M^{2}}\right)^{\frac{1}{2}}$ which is obtained from

$$
\lim _{W \rightarrow M}\left(1+\frac{Q^{2}}{(M+W)^{2}}\right)^{\frac{1}{2}}
$$

In the equations (33) and (34) there is the same common factor $\left(1+\frac{Q^{2}}{(M+W)^{2}}\right)^{\frac{1}{2}}$ and it might be natural to keep this term in order to postulate the higher resonance modification factor. By looking at the duality properties of the RS model [25] we checked that it is better to keep this factor the same as in the original FKR/RS model:

$$
\left(1+\frac{Q^{2}}{4 W^{2}}\right)^{-\frac{N}{2}} \quad \text { or } \quad\left(1+\frac{Q^{2}}{4 M^{2}}\right)^{-N}
$$

following the arguments presented in the Sect. 2. Therefore we consider two functional forms of the dependence of the $G_{V}^{R S, \text { new }}$ on the resonance oscillator levels:

$$
G_{V}^{R S, \text { new }}\left(W, Q^{2}\right)=\frac{1}{2}\left(1+\frac{Q^{2}}{(M+W)^{2}}\right)^{\frac{1}{2}}\left(1+\frac{Q^{2}}{4 W^{2}}\right)^{-\frac{N}{2}} \sqrt{3\left(G_{3}\left(W, Q^{2}\right)\right)^{2}+\left(G_{1}\left(W, Q^{2}\right)\right)^{2}}
$$

or

$$
G_{V}^{R S, \text { new }}\left(W, Q^{2}\right)=\frac{1}{2}\left(1+\frac{Q^{2}}{(M+W)^{2}}\right)^{\frac{1}{2}}\left(1+\frac{Q^{2}}{4 M^{2}}\right)^{-N} \sqrt{3\left(G_{3}\left(W, Q^{2}\right)\right)^{2}+\left(G_{1}\left(W, Q^{2}\right)\right)^{2}}
$$

with

$$
\begin{aligned}
& G_{3}\left(W, Q^{2}\right)=\frac{1}{2 \sqrt{3}}\left[C_{4}^{V} \frac{W^{2}-Q^{2}-M^{2}}{2 M^{2}}+C_{5}^{V} \frac{W^{2}+Q^{2}-M^{2}}{2 M^{2}}+\frac{C_{3}^{V}}{M}(W+M)\right] \\
& G_{1}\left(W, Q^{2}\right)=-\frac{1}{2 \sqrt{3}}\left[C_{4}^{V} \frac{W^{2}-Q^{2}-M^{2}}{2 M^{2}}+C_{5}^{V} \frac{W^{2}+Q^{2}-M^{2}}{2 M^{2}}-C_{3}^{V} \frac{(M+W) M+Q^{2}}{M W}\right]
\end{aligned}
$$

depending on the choice of an ansatz for higher $N$ behavior. We will use the parametrization (36) for inclusive $e p$ scattering and (37) for $\nu N$ scattering in agreement with the logic explained in the Sect. 4.

\subsection{Axial contribution}

The axial part of the weak CC current is:

$$
\left\langle\Delta^{++}\left(p^{\prime}\right)\left|\mathcal{J}_{\mu}^{A}\right| N(p)\right\rangle=\sqrt{3} \bar{\Psi}_{\lambda}\left(p^{\prime}\right)\left[g_{\mu}^{\lambda} B_{\nu} q^{\nu}-q^{\lambda} B_{\mu}+g_{\mu}^{\lambda} C_{5}^{A}+\frac{q^{\lambda} q_{\mu}}{M^{2}} C_{6}^{A}\right] u(p),
$$

where

$$
B_{\lambda}=\frac{C_{3}^{A}}{M} \gamma_{\lambda}+\frac{C_{4}^{A}}{M^{2}} p_{\lambda}^{\prime}
$$

The axial contributions to the $\Delta(1232)$ helicity amplitudes are calculated to be:

$$
\begin{aligned}
& f_{+3}^{\Delta, A}=-N_{q_{\text {res }}}\left\{\frac{C_{4}^{A}}{M^{2}} p^{\prime}{ }_{\mu} q^{\mu}+C_{5}^{A}+\frac{C_{3}^{A}}{M}\left(\nu_{\text {res }}+\frac{q_{\text {res }}^{2}}{M+E_{q_{r e s}}}\right)\right\}, \\
& f_{+1}^{\Delta, A}=-N_{q_{\text {res }}} \sqrt{\frac{1}{3}}\left\{\frac{C_{4}^{A}}{M^{2}} p^{\prime}{ }_{\mu} q^{\mu}+C_{5}^{A}+\frac{C_{3}^{A}}{M}\left(\nu_{\text {res }}-\frac{q_{r e s}^{2}}{M+E_{q_{r e s}}}\right)\right\}, \\
& f_{+0}^{\Delta, A}=N_{q_{r e s}} \sqrt{\frac{2}{3}}\left\{-\frac{\nu_{r e s}}{\sqrt{Q^{2}}} C_{5}^{A}+\frac{C_{3}^{A}}{M} \sqrt{Q^{2}}+\frac{C_{4}^{A}}{M^{2}} W \sqrt{Q^{2}}\right\} .
\end{aligned}
$$



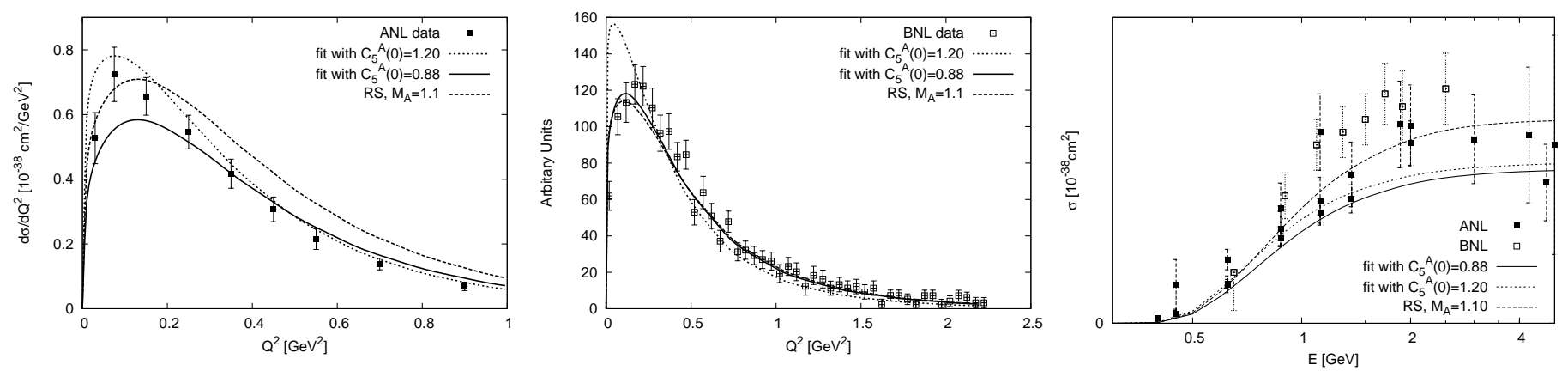

FIG. 3: Differential (left and middle figures) $d \sigma / d Q^{2}$ and total cross sections (right figure) for $\nu+p \rightarrow \mu^{-}+p+\pi^{+}$scattering. In the first figure the data (black squares) is from the ANL experiment [15]. In the second figure the data (white squares) is from the BNL experiment [16, 17]. Theoretical curves are obtained with form factors (58) where $C_{5}^{A}\left(Q^{2}\right)$ is given by (53) (solid line) and by (52) (dotted line). The cross sections calculated based on original form factors (57) with $M_{A}=1.1 \mathrm{GeV}$ are denoted by dashed lines. For the differential cross sections the cut on the invariant hadronic mass is imposed $W<1.4 \mathrm{GeV}$ whereas for the total cross sections $W<2 \mathrm{GeV}$.

In the RS model one obtains:

$$
\begin{aligned}
f_{+3}^{\Delta, A, R S} & =\sqrt{6} \sqrt{\frac{W}{M}} \frac{\sqrt{2}}{6 W}(W+M) \widetilde{G}_{A}^{R S}\left(W, Q^{2}\right) \\
f_{+1}^{\Delta, A, R S} & =\sqrt{2} \sqrt{\frac{W}{M} \frac{\sqrt{2}}{6 W}}(W+M) \widetilde{G}_{A}^{R S}\left(W, Q^{2}\right) \\
f_{+0}^{\Delta, A, R S} & =-2 \sqrt{2} \sqrt{\frac{W}{M}} \frac{1}{6 M q}\left(W^{2}-M^{2}\right) \widetilde{G}_{A}^{R S}\left(W, Q^{2}\right) \frac{q_{r e s}}{\sqrt{Q^{2}}} .
\end{aligned}
$$

In the comparison we obtain three equations which in general cannot be simultaneously satisfied.

It is natural to assume that $C_{3}^{A}=0$, because then the relation between $f_{+3}$ and $f_{+1}$ is the same in both computations:

$$
f_{+1}^{\Delta, A}=\sqrt{\frac{1}{3}} f_{+3}^{\Delta, A}
$$

In the comprehensive analysis of the $\Delta(1232)$ axial current [3, 5] the following Adler's relation is assumed:

$$
C_{4}^{A}=-\frac{1}{4} C_{5}^{A}
$$

Under this assumption the comparison of axial current helicity amplitudes leads to equations:

$$
\begin{aligned}
\widetilde{G}_{A}^{R S,+3,+1}\left(W, Q^{2}\right) & =\frac{\sqrt{3}}{2}\left(1+\frac{Q^{2}}{(M+W)^{2}}\right)^{\frac{1}{2}}\left[1-\frac{W^{2}-Q^{2}-M^{2}}{8 M^{2}}\right] C_{5}^{A}\left(Q^{2}\right), \\
\widetilde{G}_{A}^{R S,+0}\left(W, Q^{2}\right) & =\frac{\sqrt{3}}{2}\left(1+\frac{Q^{2}}{(M+W)^{2}}\right)^{\frac{1}{2}}\left[\frac{W^{2}-Q^{2}-M^{2}}{2 W(W-M)}+\frac{W Q^{2}}{4 M^{2}(W-M)}\right] C_{5}^{A}\left(Q^{2}\right) .
\end{aligned}
$$

These are two different expressions for the $\widetilde{G}_{A}^{R S, \text { new }}$. In the cross section the most important region is that of low $Q^{2}$ and the difference between them near $Q^{2}=0$ is small: $\widetilde{G}_{A}^{R S,+3,+1}\left(W=M_{\Delta}, Q^{2}=0\right)=0.945$ and $\widetilde{G}_{A}^{R S,+0}\left(W=M_{\Delta}, Q^{2}=0\right)=0.915$ (we assumed $\left.C_{5}^{A}(0)=1.2\right)$.

We tried to estimate the relative relevance of both amplitudes to the cross section but it depends on the neutrino energy and $Q^{2}$. We observed also that in the case of $\widetilde{G}_{A}^{R S,+0}$ the increase of the value of $\widetilde{G}_{A}^{R S, \text { new }}$ with $Q^{2}$ is too rapid. In order to be able to get an agreement with both sets of data we choose:

$$
\widetilde{G}_{A}^{R S, n e w}\left(W, Q^{2}\right)=\frac{\sqrt{3}}{2}\left(1+\frac{Q^{2}}{(M+W)^{2}}\right)^{\frac{1}{2}}\left[1-\frac{W^{2}-Q^{2}-M^{2}}{8 M^{2}}\right] C_{5}^{A}\left(Q^{2}\right) .
$$

We see that, under assumptions we have described, the fit to $\widetilde{G}_{A}^{R S, n e w}\left(W, Q^{2}\right)$ is equivalent to the fit to $C_{5}^{A}\left(Q^{2}\right)$. 
We define an iterative procedure to get $C_{5}^{A}\left(Q^{2}\right)$ from the data. This procedure takes into consideration differential cross sections $\left(\frac{d \sigma}{d Q^{2}}\right)_{A N L}$ measured in the ANL experiment [15] and the shape of differential cross section $\left(\frac{d \sigma}{d Q^{2}}\right)_{B N L}$ measured in the BNL experiment [17]. We use also the knowledge about neutrino fluxes in both experiments.

The fitting procedure consists of several steps:

(i) The differential cross section points for $\left(\frac{d \sigma}{d Q^{2}}\right)_{A N L}$ are translated into experimental points for $C_{A}^{5}\left(Q^{2}\right)$.

(ii) The analytical fit to obtained points is found (in order to compare with other approaches we restricted our analysis to functional forms of $C_{5}^{A}\left(Q^{2}\right)$ considered in [6]).

(iii) After the obtained fit is used to calculate the flux-averaged cross section with the BNL beam, the differential cross section points for $\left(\frac{d \sigma}{d Q^{2}}\right)_{B N L}$ are translated into experimental points for $C_{A}^{5}\left(Q^{2}\right)$.

(iv) The simultaneous fit to $C_{5}^{A}\left(Q^{2}\right)$ BNL data from point (iii) and $C_{5}^{A}\left(Q^{2}\right)$ ANL data from point (i) is found.

(v) Using the new fit from (iv) the steps (iii) and (iv) are repeated.

We define the iterative procedure. The ANL $C_{5}^{A}\left(Q^{2}\right)$ points are unchanged while each iteration moves BNL points. It was checked that the iterative procedure is quickly convergent. We needed four iteration steps to obtain $C_{A}^{5}\left(Q^{2}\right)$ which was virtually unchanged under further steps. These are the fits discussed in the remaining part of our paper. In the step (iii) one could have also started from arbitrary normalization for the BNL cross section. We checked that our fitting procedure is convergent in this case as well.

We assumed that the relevance of two data sets is the same. Since the BNL data consists of more experimental points we introduced $\geq 1$ weights to ANL points according to the number od ANL and BNL points in a given energy bin. Our final fits together with experimental points extracted from ANL and BNL experiments are shown in Fig. 1 . We notice that error bars for the BNL points for increasing $Q^{2}$ are quite large. This is because the relative significance of axial contribution is decreasing.

As explained in the introduction we obtained two fits. In the first one (case I) we keep the value $C_{A}^{5}(0)=1.2$ in accordance with the PCAC arguments. In the second fit (case II) we treat $C_{A}^{5}(0)$ as a free parameter.

Our results are:

- case I:

$$
C_{5}^{A}\left(Q^{2}\right)=\frac{C_{5}^{A}(0)}{\left(1+\frac{Q^{2}}{M_{a}^{2}}\right)^{2}}, \quad C_{5}^{A}(0)=1.2, \quad M_{a}^{2} \approx 0.54 \mathrm{GeV}^{2}
$$

- case II:

$$
C_{5}^{A}\left(Q^{2}\right)=\frac{C_{5}^{A}(0)}{\left(1+\frac{Q^{2}}{M_{a}^{2}}\right)^{2}\left(1+\frac{Q^{2}}{M_{b}^{2}}\right)}, \quad C_{5}^{A}(0) \approx 0.88, \quad M_{a}^{2} \approx 9.71 \mathrm{GeV}^{2}, \quad M_{b}^{2} \approx 0.35 \mathrm{GeV}^{2}
$$

Finally, we define the generalization of $\widetilde{G}_{A}^{R S, n e w}$ for higher $N$ along the lines explained before and we obtain:

$$
\widetilde{G}_{A}^{R S, \text { new }}\left(W, Q^{2}\right)=\frac{\sqrt{3}}{2}\left(1+\frac{Q^{2}}{(M+W)^{2}}\right)^{\frac{1}{2}}\left(1+\frac{Q^{2}}{4 M^{2}}\right)^{-N}\left[1-\frac{W^{2}-Q^{2}-M^{2}}{8 M^{2}}\right] C_{5}^{A}\left(Q^{2}\right) .
$$



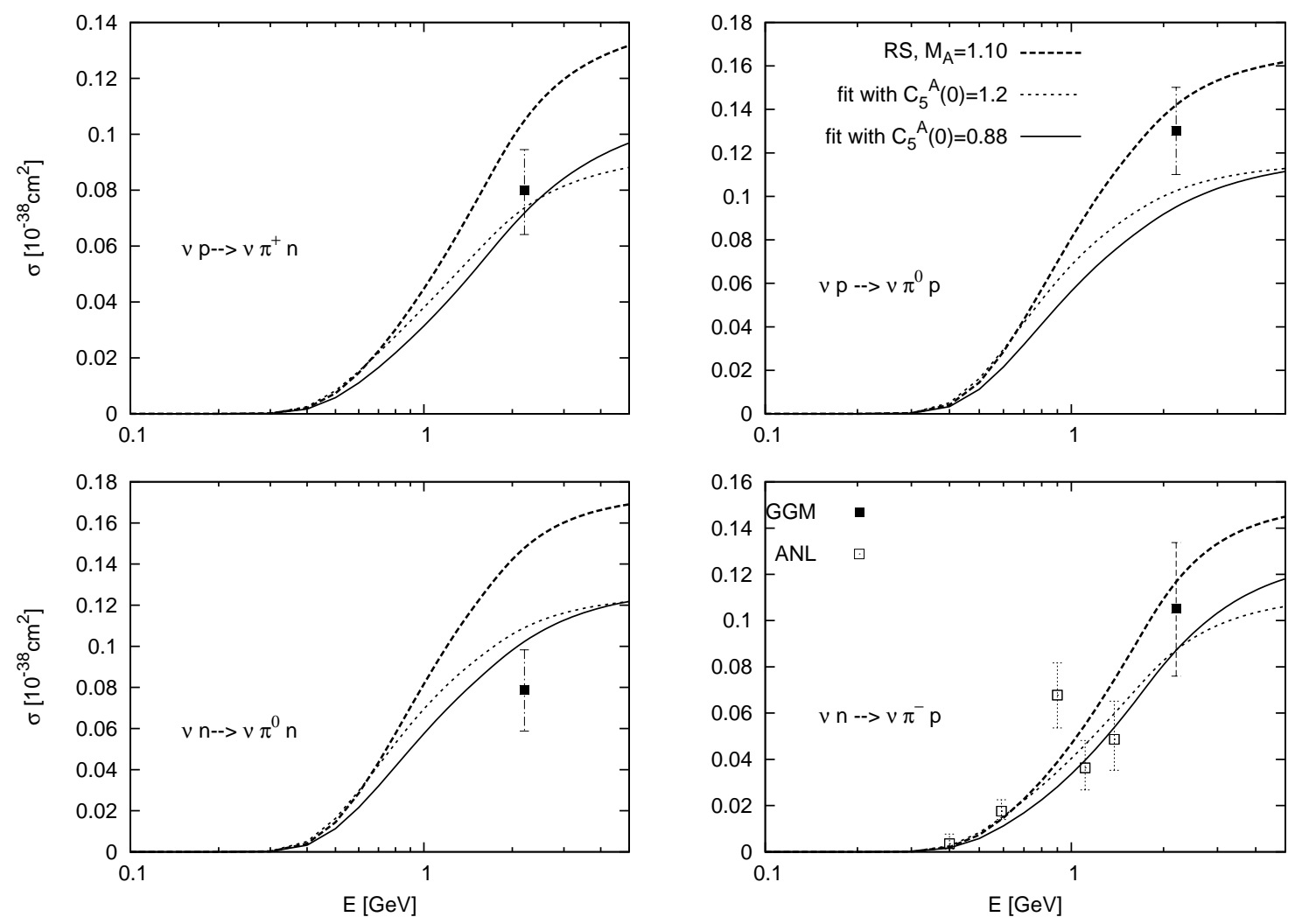

FIG. 4: Total cross sections for SPP in NC neutrino-nucleon scattering. The data is from the experiments: GGM [34] (black squares) and ANL [35] (white squares). Theoretical curves are obtained with form factors (58) and $C_{5}^{A}\left(Q^{2}\right)$ given by (53) (solid line) or by (52) (dotted line). The cross sections calculated based on the original RS form factors (57) with $M_{A}=1.1 \mathrm{GeV}$ are denoted by dashed lines. The cut on the invariant hadronic mass $W<2 \mathrm{GeV}$ is imposed.

\section{DISCUSSION}

In Fig. 2 (top plots) we show predictions of the FKR model for the electroproduction. In this case the precise data exist for the inclusive $F_{2}$ proton structure function [24]. In the theoretical computation contributions from 18 resonances (taken form [10]) are calculated.

We compare predictions based on the following parameterizations of $G_{V}$ :

$$
G_{V}^{R S}\left(W, Q^{2}\right)=\left(1+\frac{Q^{2}}{4 W^{2}}\right)^{\frac{1-N}{2}}\left(1+\frac{Q^{2}}{M_{V}^{2}}\right)^{-2}
$$

and

$$
G_{V}^{R S, \text { new }}\left(W, Q^{2}\right)=\frac{1}{2}\left(1+\frac{Q^{2}}{(M+W)^{2}}\right)^{\frac{1}{2}}\left(1+\frac{Q^{2}}{4 W^{2}}\right)^{-\frac{N}{2}}\left[3\left(G_{3}\left(W, Q^{2}\right)\right)^{2}+\left(G_{1}\left(W Q^{2}\right)\right)^{2}\right]^{\frac{1}{2}} .
$$

It is seen that for both values $Q^{2}=0.225 \mathrm{GeV}^{2}$ and $Q^{2}=1.025 \mathrm{GeV}^{2}$ the results with the new vector form factor are closer to experimental data. The large difference is seen in particular in the $\Delta(1232)$ resonance region.

At the $\Delta(1232)$ resonance peak some strength is missing, also with the new form factors, and a non-resonant dynamics is believed to be responsible for that. In the Fig. 2 (bottom figures) we show the evaluation of the ratio of the proton $F_{2}$ calculated within the FKR model (only resonance contribution) and the experimental data. The computations are done for both form factors. At the $\Delta(1232)$ resonance peak with the new vector form factor the missing strength is $10 \div 20 \%$ depending on the value of $Q^{2}$. The similar relative contribution (about $25 \%$ ) of the background dynamics is seen also in plots presented in [36]. 


\begin{tabular}{|c|c|c|}
\hline \hline $\begin{array}{c}\text { Electromagnetic } \\
\text { Helicity Amplitudes }\end{array}$ & Standard Approach & RS model \\
\hline$f_{+0}^{e m, p}$ & $\left(1+\frac{Q^{2}}{4 M^{2}}\right)^{-\frac{1}{2}} G_{E}^{p}\left(Q^{2}\right)$ & $\left(1-\frac{Q^{2}}{2 M^{2}}\right)\left(1+\frac{Q^{2}}{4 M^{2}}\right)^{-1} G_{V}^{R S}\left(W, Q^{2}\right)$ \\
$f_{+1}^{e m, p}$ & $\frac{q}{\sqrt{2} M}\left(1+\frac{Q^{2}}{4 M^{2}}\right)^{-\frac{1}{2}} G_{M}^{p}\left(Q^{2}\right)$ & $3 \frac{q}{\sqrt{2} M}\left(1+\frac{Q^{2}}{4 M^{2}}\right)^{-1} G_{V}^{R S}\left(W, Q^{2}\right)$ \\
$f_{+0}^{e m, n}$ & $\left(1+\frac{Q^{2}}{4 M^{2}}\right)^{-\frac{1}{2}} G_{E}^{n}\left(Q^{2}\right)$ & 0 \\
$f_{+1}^{e m, n}$ & $\frac{q}{\sqrt{2} M}\left(1+\frac{Q^{2}}{4 M^{2}}\right)^{-\frac{1}{2}} G_{M}^{n}\left(Q^{2}\right)$ & $-2 \frac{q}{\sqrt{2} M}\left(1+\frac{Q^{2}}{4 M^{2}}\right)^{-1} G_{V}^{R S}\left(W, Q^{2}\right)$ \\
\hline
\end{tabular}

TABLE I: The elastic electromagnetic helicity amplitudes.

\begin{tabular}{|c|c|c|}
\hline \hline CC Helicity Amplitudes & Standard Approach [37] & RS model \\
\hline$f_{+0}^{A}$ & 0 & 0 \\
$f_{+1}^{A}$ & $\sqrt{2}\left(1+\frac{Q^{2}}{4 M^{2}}\right)^{\frac{1}{2}} G_{A}\left(Q^{2}\right)$ & $\frac{5 \sqrt{2}}{3} Z G_{A}^{R S}\left(Q^{2}\right)$ \\
$f_{+0}^{V}$ & $\left(1+\frac{Q^{2}}{4 M^{2}}\right)^{-\frac{1}{2}}\left(G_{E}^{p}\left(Q^{2}\right)-G_{E}^{n}\left(Q^{2}\right)\right)$ & $\left(1-\frac{Q^{2}}{2 M^{2}}\right)\left(1+\frac{Q^{2}}{4 M^{2}}\right)^{-1} G_{V}^{R S}\left(Q^{2}\right)$ \\
$f_{+1}^{V}$ & $\frac{q}{\sqrt{2} M}\left(1+\frac{Q^{2}}{4 M^{2}}\right)^{-\frac{1}{2}}\left(G_{M}^{p}\left(Q^{2}\right)-G_{M}^{n}\left(Q^{2}\right)\right)$ & $5 \frac{q}{\sqrt{2} M}\left(1+\frac{Q^{2}}{4 M^{2}}\right)^{-1} G_{V}^{R S}\left(Q^{2}\right)$ \\
\hline
\end{tabular}

TABLE II: The quasi-elastic weak CC helicity amplitudes.

In $\mathrm{CC}$ neutrino-production of resonances vector and axial parts of the weak current are tested simultaneously. We compare predictions based on two different sets of form factors. In the first one:

$$
G_{V}^{R S}=\left(1+\frac{Q^{2}}{4 M^{2}}\right)^{\frac{1}{2}-N}\left(1+\frac{Q^{2}}{M_{V}^{2}}\right)^{-2}, \quad \widetilde{G}_{A}^{R S}\left(Q^{2}\right)=0.76\left(1+\frac{Q^{2}}{4 M^{2}}\right)^{\frac{1}{2}-N}\left(1+\frac{Q^{2}}{M_{A}^{2}}\right)^{-2}
$$

and in the second one:

$$
G_{V}^{R S, n e w}\left(W, Q^{2}\right)=\frac{1}{2}\left(1+\frac{Q^{2}}{(M+W)^{2}}\right)^{\frac{1}{2}}\left(1+\frac{Q^{2}}{4 M^{2}}\right)^{-N}\left[3\left(G_{3}\left(W, Q^{2}\right)\right)^{2}+\left(G_{1}\left(W, Q^{2}\right)\right)^{2}\right]^{\frac{1}{2}} .
$$




\begin{tabular}{|c|c|c|}
\hline \hline Form Factors & Proton & Neutron \\
\hline$G_{E}\left(Q^{2}\right)$ & $\left(1-\frac{Q^{2}}{2 M^{2}}\right)\left(1+\frac{Q^{2}}{M_{V}^{2}}\right)^{-2}$ & 0 \\
\hline$G_{M}\left(Q^{2}\right)$ & $3\left(1+\frac{Q^{2}}{M_{V}^{2}}\right)^{-2}$ & $-2\left(1+\frac{Q^{2}}{M_{V}^{2}}\right)^{-2}$ \\
\hline
\end{tabular}

\begin{tabular}{|c|c|}
\hline \hline \multicolumn{2}{|c|}{ Axial form factor } \\
\hline$G_{A}\left(Q^{2}\right)$ & $\frac{5}{3} Z\left(1+\frac{Q^{2}}{M_{A}^{2}}\right)^{-2}$ \\
\hline \hline
\end{tabular}

TABLE III: In the top proton and neutron electric and magnetic elastic form factors obtained within the RS model are shown. In the bottom the axial nucleon form factor obtained within the RS model is presented $\left(\frac{5}{3} Z \approx 1.267\right)$.

$$
\widetilde{G}_{A}^{R S, \text { new }}\left(W, Q^{2}\right)=\frac{\sqrt{3}}{2}\left(1+\frac{Q^{2}}{(M+W)^{2}}\right)^{\frac{1}{2}}\left(1+\frac{Q^{2}}{4 M^{2}}\right)^{-N}\left[1-\frac{W^{2}-Q^{2}-M^{2}}{8 M^{2}}\right] C_{5}^{A}\left(Q^{2}\right) .
$$

The first set was used in the original RS paper. As was shown in Sect. 3 according to the logic of the RS model $M_{A}$ should be the axial mass parameter of the quasi-elastic neutrino scattering. But usually $M_{A}$ is considered as a free parameter fitted with the help of neutrino SPP data. The measurements of $M_{A}$ give values around $1.00 \mathrm{GeV}$ [27]. However, recent K2K [28] and MiniBooNE investigations [29] indicate that the value of $M_{A}$ can be as big as $1.2 \mathrm{GeV}$. In this paper we show the predictions of the RS model with the axial mass $M_{A}=1.1 \mathrm{GeV}[32$ ].

In the computations with the original RS form factors we take into account the normalization factors $\mathcal{C}_{\mathcal{N} *}$ introduced in [10] coming from the Breit-Wigner amplitudes:

$$
\delta\left(W-M_{R}\right) \rightarrow \sqrt{\frac{\Gamma(W)}{2 \pi}} \frac{1}{W-M_{R}+\mathrm{i} \Gamma(W) / 2} \cdot \frac{1}{\sqrt{\mathcal{C}_{\mathcal{N}^{*}}}},
$$

where

$$
\mathcal{C}_{\mathcal{N}^{*}} \equiv \int_{W_{\text {thr }}}^{\infty} d W \frac{\Gamma(W)}{2 \pi} \frac{1}{\left(W-M_{R}\right)^{2}+(\Gamma(W))^{2} / 4}
$$

and $W_{t h r}=M+m_{\pi} \approx 1.08 \mathrm{GeV}$ is the threshold for SPP. For the $\Delta(1232)$ resonance: $\mathcal{C}_{\Delta} \approx 0.87$ and for higher resonances $\mathcal{C}_{\mathcal{N}^{*}}$ range from 0.75 to 1.30 . In computations with new form factors we do not include $\mathcal{C}_{\mathcal{N}^{*}}$ because they are not present in phenomenological Rarita Schwinger formalism for $\Delta(1232)$ excitation [37].

In numerical analysis for neutrino-nucleon interaction we use the RS approach with lepton mass effects as it is described in [26].

In Fig. 3 we compare predictions of RS model with the experimental results for $\frac{d \sigma}{d Q^{2}}$ and total cross section for $\nu+p \rightarrow \mu^{-}+\Delta^{++}(1232)$. This reaction is most suitable to discuss because the non-resonant contribution in the $\Delta(1232)$ region is small [15]. We use the data from ANL [15] and BNL [16, 17] experiments. The ANL energy beam distribution ranges from $0 \div 3 \mathrm{GeV}$ and has a peak at $E \simeq 0.9 \mathrm{GeV}$. The BNL energy beam distribution ranges from $0 \div 6 \mathrm{GeV}$ and the peak is at $E \simeq 1.2 \mathrm{GeV}$. In the case of ANL data the differential cross section is normalized to the actual cross section and the BNL data are given in arbitrary units so that only the shape of $\frac{d \sigma}{d Q^{2}}$ is relevant.

We see that predictions of our model with $C_{5}^{A}(0)=0.88$ agree well with both sets of points. The model with $C_{5}^{A}(0)=1.2$ agrees with ANL data but overestimates BNL data at low $Q^{2}$.

We investigated also the relevance of new form factors for the prediction of cross sections for NC single pion production (see Fig. (4). In this case only few experimental points exist. The modification of the form factors changes the predictions of the RS model in the significant way. 


\section{CONCLUSIONS}

We proposed new vector and axial form factors which should improve the performance of the RS model in the $\Delta(1232)$ resonance region. In the case of axial form factor we consider a simultaneous fit to both ANL and BNL sets of data without introduction of background terms. Our best fit corresponds to $C_{5}^{A}(0) \approx 0.88$. Our results are based on assumptions specific for the RS model and it would be interesting to check if the same can be done in the Rarita Schwinger formalism. Before it was claimed that separate fits must be applied to agree with either ANL or BNL data [6].

\section{Acknowledgements}

The authors were supported by the KBN grant 3735/H03/2006/31. JTS thanks Olga Lalakulich for an information about the paper [24].

\section{APPENDIX A}

The quantities to calculate are helicity amplitudes:

$$
f_{+1}^{e m, N} \equiv(2 \pi)^{3} \sqrt{\frac{E_{p, r e s}}{M}}\left\langle N, s^{\prime}=\frac{1}{2}\left|\mathcal{J}_{+}^{e m}\right| N, s=-\frac{1}{2}\right\rangle, \quad f_{+0}^{e m, N} \equiv(2 \pi)^{3} \sqrt{\frac{E_{p, r e s}}{M}}\left\langle N, s^{\prime}=\frac{1}{2}\left|\mathcal{J}_{\underline{0}}^{e m}\right| N, s=\frac{1}{2}\right\rangle,
$$

where $\mathrm{N}=p$ or $n$ denotes nucleon target.

For the CC neutrino-nucleon scattering we need to compute vector and axial transition matrix elements:

$$
f_{+1}^{V, A} \equiv(2 \pi)^{3} \sqrt{\frac{E_{p, r e s}}{M}}\left\langle p, s^{\prime}=\frac{1}{2}\left|\mathcal{J}_{+}^{V, A}\right| n, s=-\frac{1}{2}\right\rangle, \quad f_{+0}^{V, A} \equiv(2 \pi)^{3} \sqrt{\frac{E_{p, \text { res }}}{M}}\left\langle p, s^{\prime}=\frac{1}{2}\left|\mathcal{J}_{\underline{0}}^{V, A}\right| n, s=\frac{1}{2}\right\rangle .
$$

In the case of elastic electron-proton scattering the transition matrix elements are:

$$
f_{e m, p}^{\mu}\left(s^{\prime}, s\right)=\bar{u}\left(p^{\prime}, s^{\prime}\right)\left(F_{1}^{e m, p}\left(Q^{2}\right) \gamma^{\mu}+\frac{i \sigma^{\mu \nu} q_{\nu}}{2 M} F_{2}^{e m, p}\left(Q^{2}\right)\right) u(p, s) .
$$

$f_{\mu}$ are computed in the rest frame of the final nucleon. The Dirac spinors for the incoming and outgoing nucleons are:

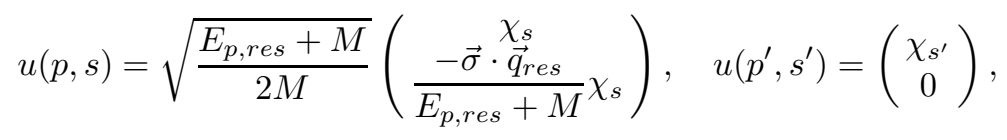

where $\chi_{s}, \chi_{s^{\prime}}$ are 2-component spinors.

The relevant combinations of the current (A1) give rise to:

$$
\begin{aligned}
& f_{\underline{0}}^{e m, p}\left(s^{\prime}, s\right)=\chi_{s^{\prime}}^{\dagger} G_{E}^{e m, p}\left(1+\frac{Q^{2}}{4 M^{2}}\right)^{-\frac{1}{2}} \chi_{s}, \\
& f_{ \pm}^{e m, p}\left(s^{\prime}, s\right)=\mp \frac{1}{\sqrt{2}}\left(f_{1}\left(s^{\prime}, s\right) \pm i f_{2}\left(s^{\prime}, s\right)\right)=\chi_{s^{\prime}}^{\dagger} \frac{q \sigma_{ \pm}}{\sqrt{2} M} G_{M}^{e m, p}\left(1+\frac{Q^{2}}{4 M^{2}}\right)^{-\frac{1}{2}} \chi_{s}
\end{aligned}
$$

so that

$$
\begin{aligned}
& f_{+0}^{e m, p} \equiv f_{\underline{0}}(1 / 2,1 / 2)=G_{E}^{e m, p}\left(1+\frac{Q^{2}}{4 M^{2}}\right)^{-\frac{1}{2}} \\
& f_{+1}^{e m, p} \equiv f_{+}(1 / 2,-1 / 2)=\frac{q}{\sqrt{2} M} G_{M}^{e m, p}\left(1+\frac{Q^{2}}{4 M^{2}}\right)^{-\frac{1}{2}} .
\end{aligned}
$$

The helicity amplitudes for the electron-neutron scattering are obtained by substitution in (A3) $G_{E, M}^{p} \rightarrow G_{E, M}^{n}$. 
Similar computations are done for the quasi-elastic CC neutrino-neutron scattering:

$$
\begin{aligned}
& f^{\mu}\left(s^{\prime}, s\right)=f_{V}^{\mu}\left(s^{\prime}, s\right)-f_{A}^{\mu}\left(s^{\prime}, s\right) \\
& f_{V}^{\mu}\left(s^{\prime}, s\right)=\bar{u}\left(p^{\prime}, s^{\prime}\right)\left(F_{1}\left(Q^{2}\right) \gamma^{\mu}+\frac{i \sigma_{\mu \nu} q^{\nu}}{2 M} F_{2}\left(Q^{2}\right)\right) u(p, s), \\
& f_{A}^{\mu}\left(s^{\prime}, s\right)=-\bar{u}\left(p^{\prime}, s^{\prime}\right)\left(\gamma^{\mu} \gamma_{5} G_{A}\left(Q^{2}\right)+q^{\mu} \gamma_{5} F_{P}\left(Q^{2}\right)\right) u(p, s) .
\end{aligned}
$$

The vector part of the above current is the same as in the electromagnetic interactions and to get the matrix elements it is enough to make a replacement $G_{E, M}^{p} \rightarrow G_{E, M}^{p}-G_{E, M}^{n}$.

The axial part results are:

$$
f_{+0}^{A}=0, \quad f_{+1}^{A}=\sqrt{2}\left(1+\frac{Q^{2}}{4 M^{2}}\right)^{\frac{1}{2}} G_{A} .
$$

Analogous calculations, must be done in the Rein-Sehgal model. Hadronic currents are operators expressed in terms of spin $\sigma_{a}$, isospin $\tau_{a}$ quark operators and annihilation, creation $\left(a, a^{\dagger}\right)$ operators from the 3-dimensional harmonic oscillator (for detailed explanation see e.g. [12]).

The vector components of the hadronic currents read:

$$
\mathcal{J}_{\underline{0}}^{V}=9 \tau_{a}^{+} e^{-\lambda a^{z \dagger}} S, \quad \mathcal{J}_{ \pm}^{V}=9 \tau_{a}^{+} e^{-\lambda a^{z \dagger}}\left(T_{V} a_{ \pm}^{\dagger}+R_{V} \sigma^{ \pm}\right),
$$

where

$$
S=\frac{Q^{2}}{q_{\text {res }}^{2}} \frac{3 W M-Q^{2}-M^{2}}{3 W} G_{V}^{R S}, \quad T_{V}=\frac{2}{3} \sqrt{\frac{\Omega}{2}} G_{V}^{R S}, \quad R_{V}=\sqrt{2} \frac{2 W q_{r e s}(M+W)}{(M+W)^{2}+Q^{2}} G_{V}^{R S} .
$$

The axial current is expressed as:

$$
\mathcal{J}_{\underline{0}}^{A}=-9 \tau_{a}^{+} e^{-\lambda a^{z \dagger}}\left(C \sigma_{a}^{3}+B \vec{\sigma}_{a} \cdot \vec{a}^{\dagger}\right), \quad \mathcal{J}_{ \pm}^{A}= \pm 9 \tau_{a}^{ \pm} e^{-\lambda a^{z \dagger}}\left[R_{A} \sigma_{a}^{+}+T_{A} a_{ \pm}^{\dagger}\right]
$$

where

$$
\begin{gathered}
B=G_{A}^{R S} \frac{2 Z}{3} \sqrt{\frac{\Omega}{2}}\left[1+\frac{W^{2}-Q^{2}-M^{2}}{(W+M)^{2}+Q^{2}}\right], \quad C=G_{A}^{R S} \frac{Z W}{3 M q}\left[W^{2}-M^{2}+N \Omega \frac{W^{2}-Q^{2}-M^{2}}{(W+M)^{2}+Q^{2}}\right], \\
R_{A}=Z G_{A}^{R S} \frac{\sqrt{2}}{3}\left(W+M+\frac{2 N \Omega W}{(W+M)^{2}+Q^{2}}\right), \quad T_{A}=\frac{4}{3} Z \sqrt{\frac{\Omega}{2}} \frac{q M}{(W+M)^{2}+Q^{2}} G_{A}^{R S} \sqrt{\frac{\Omega}{2}}
\end{gathered}
$$

$\lambda=\sqrt{\frac{2}{\Omega}} q_{\text {res }}, \Omega=1.05 \mathrm{GeV}^{2}$ is determined from the Regge slope of baryon trajectories, $N$ is the oscillator level of a given resonance.

In the quark model the matrix elements of $\tau_{a}^{+}$and $\tau_{a}^{+} \sigma_{a}^{ \pm}$(acting on the first quark) are [20]:

$$
\left\langle p, \frac{1}{2}\left|\tau_{a}^{+}\right| n, \frac{1}{2}\right\rangle=\frac{1}{3}, \quad\left\langle p, \frac{1}{2}\left|\tau_{a}^{+} \sigma_{+}\right| n,-\frac{1}{2}\right\rangle=\frac{5}{9} .
$$

Therefore:

$$
f_{+0}^{V}=\left(1+\frac{Q^{2}}{4 M^{2}}\right)^{-1}\left(1-\frac{Q^{2}}{2 M^{2}}\right) G_{V}^{R S}, \quad f_{+1}^{V}=5 \frac{q}{\sqrt{2} M}\left(1+\frac{Q^{2}}{4 M^{2}}\right) G_{V}^{R S}, \quad f_{+0}^{A}=0, \quad f_{+1}^{A}=Z_{\frac{5 \sqrt{2}}{3}} G_{A}^{R S} .
$$

The outcome of computation is summarized in Tabs. III where we collect helicity amplitudes computed in elastic $e N$ and quasi-elastic $\nu n$ scattering. If we assume that the vector and axial form factors of the RS model are

$$
G_{V}^{R S}=\left(1+\frac{Q^{2}}{4 M^{2}}\right)^{\frac{1}{2}}\left(1+\frac{Q^{2}}{M_{V}^{2}}\right)^{-2}, \quad G_{A}^{R S}=\left(1+\frac{Q^{2}}{4 M^{2}}\right)^{\frac{1}{2}}\left(1+\frac{Q^{2}}{M_{A}^{2}}\right)^{-2}
$$

the electric, magnetic and axial nucleon form factors take a familiar form shown in Tab. III. 
[2] S. L. Adler, Annals Phys. 50 (1968) 189.

[3] P. A. Schreiner and F. Von Hippel, Nucl. Phys. B 58 (1973) 333.

[4] G. L. Fogli and G. Nardulli, Nucl. Phys. B 160 (1979) 116.

[5] L. Alvarez-Ruso, S. K. Singh and M. J. Vincente Vascas, Phys. Rev. 57 (1998) 2693.

[6] O. Lalakulich and E. A. Paschos, Phys. Rev. D 71 (2005) 074003.

[7] T. Sato, D. Uno and T. S. H. Lee, Phys. Rev. C 67 (2003) 065201.

[8] E. Hernandez, J. Nieves, and M. Valverde, Phys. Rev. D 76 (2007) 033005.

[9] F. Ravndal, Nuovo Cimento, 18A 385 (1973).

[10] D. Rein and L. M. Sehgal, Annals Phys. 133 (1981) 79.

[11] D. Rein, Z. Phys. C 35 (1987) 43.

[12] R.P. Feynman, M. Kislinger, and F. Ravndal, Phys. Rev. D 3, 2706 (1971).

[13] O. Lalakulich, E. A. Paschos and G. Piranishvili, Phys. Rev. D 74 (2006) 014009.

[14] D. Drechsel, O. Hanstein, S. S. Kamalov, and L. Tiator, Nucl. Phys. A 645 (1999) 145.

[15] G. M. Radecky et al., Phys. Rev. D 25 (1982) 1161.

[16] T. Kitagaki et al., Phys. Rev. D 34 (1986) 2554.

[17] T. Kitagaki et al., Phys. Rev. D 42 (1990) 1331.

[18] C. Alexandrou, T. Leontiou, J. W. Negele and A. Tsapalis, Phys. Rev. Lett. 98 (2007) 052003.

[19] D. Barquilla-Cano, A. J. Buchmann, and E. Hernandez, Phys. Rev. C 75 (2007) 065203.

[20] For a general review see e.g. F. E. Close, An introduction to Quarks and Partons, Academic Press 1979 Inc. (London); A. J. G. Hey and R. L. Kelly, Phys. Rept. 96 (1983) 71; G. Karl, Int. J. Mod. Phys. E 1 (1992) 491.

[21] F. Ravndal, A relativistic quark model with harmonic dynamics, PhD. Thesis, California Institute of Technology, Pasadena, California 1971.

[22] F. Ravndal, Lett. Nuovo Cimento, 3, 631 (1972).

[23] F. Ravndal, Phys. Rev. D 4, 1466 (1971).

[24] M. Osipenko et al., The proton structure function F2 in the resonance region, Published in *Genova 2002, GerasimovDrell-Hearn sum rule and the spin structure of the nucleon* 265-280. arXiv:hep-ex/0301033

[25] K. M. Graczyk, C. Juszczak, and J. T. Sobczyk, Nucl. Phys. A 781 (2007) 227.

[26] K. M. Graczyk and J. T. Sobczyk, Lepton mass effects in weak CC single pion production, arXiv:0709.4634 [hep-ph].

[27] H. Budd, A. Bodek, and J. Arrington, Nucl. Phys. Proc. Suppl. 139 (2005) 90.

[28] R. Gran et al. [K2K Collaboration], Phys. Rev. D 74 (2006) 052002.

[29] A. A. Aguilar-Arevalo et al. [MiniBooNE Collaboration], arXiv:0706.0926 [hep-ex].

[30] M. H. Ahn, et al. [K2K Collaboration], Phys. Rev. D74, 072003 (2006).

[31] http://www-boone.fnal.gov/ [MiniBooNE Collaboration].

[32] K. Furuno et al., BNL 7-foot bubble chamber experiment: Neutrino deuterium interactions, Prepared for 2nd International Workshop on Neutrino - Nucleus Interactions in the Few GeV Region (NUINT 02), Irvine, California, $12-15$ Dec 2002.

[33] J. Campbell et al., Phys. Rev. Lett. 30 (1973) 335; S. J. Barish et al., Phys. Rev. D 19 (1979) 2521.

[34] W. Krenz et al. [Gargamelle Neutrino Propane Collaboration], Nucl. Phys. B 135 (1978) 45.

[35] M. Derrick et al., Phys. Lett. B 92 (1980) 363 [Erratum-ibid. B 95 (1980) 461].

[36] H. Nakamura, M. Sakuda, T. Nasu, and O. Benhar, Inclusive electron spectrum in the region of pion production in electronnucleus scattering and the effect of the quasi-elastic interaction, arXiv:0705.3884 [hep-ph].

[37] C. H. Llewellyn Smith, Phys. Rept. 3 (1972) 261. 